\title{
Estudio comparativo del comportamiento de movimiento de 24 horas, en estudiantes universitarios de pedagogía en educación física 24-hour movement behaviors of university students of pedagogy in physical education. Comparative study by gender, between cohorts \\ *Andrés Godoy-Cumillaf, *Paola Fuentes-Merino, **Judith Jiménez-Díaz, ***Jaime Vásquez-Gómez *Universidad Autónoma de Chile (Chile), **Universidad de Costa Rica (Costa Rica), ***Universidad Católica del Maule
} (Chile)

Resumen. Investigaciones demuestran que estudiantes universitarios de Pedagogía en Educación Física presentan mejores parámetros de condición física, sin embargo, es poca la información disponible sobre comportamiento de movimiento de 24 horas, medidas de manera objetiva. El objetivo de la presente investigación es describir conducta sedentaria, AF y patrones de sueño; determinar si existen diferencias significativas entre estudiantes según sexo y año que se encuentran cursando en la universidad; y determinar si existe asociación entre intensidad de AF y patrones de sueño. Se trabajó con una muestra de 128 estudiantes, en los cuales se evaluó de manera objetiva conducta sedentaria, AF de intensidad ligera, moderada y vigorosa, y patrones del sueño (tiempo de sueño, tiempo en cama y eficiencia del sueño). Los resultados indican que los estudiantes de primer año presentan para las tres intensidades de $\mathrm{AF}$, los valores más altos de minutos promedio por día (hombres: ligera $=$ $71.5 \mathrm{~min}$, moderada $=100.4 \mathrm{~min}$, vigorosa $=12 \mathrm{~min}$; mujeres: ligera $=80.3 \mathrm{~min}$, moderada $=93.8 \mathrm{~min}$, vigorosa $=6.5 \mathrm{~min})$, además de presentar menor tiempo de sueño, sin embargo, su eficiencia del sueño es más alta. En conclusión, los estudiantes de primer año presentan menor tiempo de conducta sedentaria, mayor cantidad de AF en las tres intensidades y menor tiempo de sueño, pero mayor eficiencia. Al comparar por sexo, las mujeres presentan menor cantidad de AF de intensidad moderada y vigorosa. AF de intensidad ligera se asoció con los patrones del sueño evaluados.

Palabras claves: acelerómetros, intensidad, eficiencia, minutos.

Abstract. Research shows that university students of Pedagogy in Physical Education present better parameters of physical condition, however, little information is available on sedentary behavior, physical activity (PA) and sleep patterns measured objectively. The objective of this research is to describe sedentary behavior, PA and sleep patterns; determine if there are significant differences between students according to sex and year of admission to university; and determine if there is an association between PA intensity and sleep patterns. We worked with a sample of 128 students, in which sedentary behavior, PA of light, moderate and vigorous intensity, and sleep patterns (sleep time, time in bed and sleep efficiency) were objectively evaluated. The results indicate that first year students present the highest values of average minutes per day for the three PA intensities (men: light $=71.5 \mathrm{~min}$, moderate $=100.4 \mathrm{~min}$, vigorous $=12 \mathrm{~min}$; women: light $=80.3 \mathrm{~min}$, moderate $=93.8$ $\min$, vigorous $=6.5 \mathrm{~min}$ ), in addition to having less sleep time, however, their sleep efficiency is higher. In conclusion, firstyear students present less time of sedentary behavior, greater amount of PA in the three intensities and less time of sleep, but greater efficiency. When comparing by sex, women present less PA of moderate and vigorous intensity. Light intensity PA was associated with assessed sleep patterns.

Key words: accelerometer, intensity, efficiency, minutes.

\section{Introducción}

Diversas investigaciones sugieren que los estudiantes universitarios de Pedagogía en Educación Física presentan mejores parámetros de condición física que sus pares de otras carreras universitarias (Almagià, Lizana, Rodríguez, Ivanovich, \& Binvignat, 2009; Durán, Valdés, Godoy, \& Herrera, 2014; Valdés-Badilla, Godoy-

Fecha recepción: 20-01-21. Fecha de aceptación: 01-07-21

Andrés Godoy Cumillaf

andres.godoy@uautonoma.cl
Cumillaf, Herrera-Valenzuela, \& Durán-Agüero, 2016), sin embargo, se evidencia una disminución a medida que avanzan en sus años de formación, es así como estudiantes que se encuentran en su primer año de universidad, presentan valores más altos para resistencia cardiorrespiratoria y fuerza muscular que aquellos que cursan cuarto año. Disminución ocasionada principalmente a que la rutina universitaria provoca una falta de tiempo para realizar ejercicio físico (Durán et al., 2014; Valdés-Badilla, Godoy-Cumillaf, \& Gedda-Muñoz, 2013).

En lo que respecta a conducta sedentaria y actividad física (AF), la evidencia muestra que después de cuatro 
años de universidad, los estudiantes de pedagogía en Educación Física presentan una disminución de la cantidad de AF realizada para todas sus intensidades y un aumento de las conductas sedentarias (Vásquez-Gómez et al., 2018), sin embargo esta información debe ser analizada con prudencia, ya que fue recopilada a través de cuestionario auto-reportado, lo que puede provocar una subestimación de los valores reales (Aguilar-Farías \& Leppe, 2016).

Para obtener información objetiva de estas variables se utiliza como instrumento el acelerómetro, el cual ha reportado en estudiantes universitarios de diversas carreras gran cantidad de conductas sedentarias (Rodríguez-Rodríguez, Cristi-Montero, Villa-González, Solís-Urra \& Chillón, 2018), sin embargo, se desconoce de estudios realizados en Chile que evalúen a los estudiantes de Pedagogía en Educación Física utilizando este instrumento, lo que no permite corroborar la evidencia ya existente, determinar posibles diferencias entre estudiantes según los años que llevan en la universidad, o saber si cumplen con las recomendaciones mundiales de actividad física para las personas de su edad (i.e. un mínimo de entre 150 y 300 minutos de AF moderada, o bien un mínimo de entre 75 y 150 minutos de AF vigorosa, o una combinación equivalente de AF moderada y vigorosa) (Organización Mundial para la Salud, 2020).

Otro elemento a considerar es el sueño, el cual se define como un estado fisiológico de conciencia reducida, ausencia de actividad voluntaria y suspensión de actividad sensorial (Durán-Agüero et al., 2016), el cual es necesario para mantener en óptimas condiciones la memoria y las funciones cognitivas, motoras y metabólicas (Diekelmann \& Born, 2010; Lazar et al., 2012). Investigaciones que han estudiado el sueño en universitarios, han observado que es común en esta población presentar dificultad para dormir (Grieser et al., 2011; Peltzer \& Pengpid, 2015), falta de sueño (Lund, Reider, Whiting, \& Prichard, 2010), trastornos del sueño, síndrome de fase retardada del sueño y somnolencia diurna excesiva (Wolfson, 2010), lo que se ocasiona principalmente debido al estrés que genera el ambiente académico (Lund et al., 2010). Estudios con población universitaria de Chile, en los que se han utilizado cuestionarios para recopilar la información, muestran que presentan altos niveles de somnolencia e insomnio, concluyendo que es frecuente en ellos la mala calidad del sueño (Durán-Agüero etal., 2016; Durán, Rosales, Moya \& García, 2017; Vélez et al., 2013). No se han encontrado investigaciones en el tema con los estudiantes universitarios de Pedagogía en Educación Física.
En lo que respecta a la relación sueño y AF, se considera que ambos se influyen mutuamente, aunque este vínculo depende de algunos factores donde se encuentra la intensidad del ejercicio (Youngstedt \& Kline, 2006; Chennaoui, Arnal, Sauvet, \& Léger, 2015; Kelley \& Kelley, 2017). En este sentido, investigaciones que han estudiado la relación intensidad del ejercicio con sueño no han logrado generar un consenso acerca de la que trae los mejores beneficios (Driver \& Taylor, 2000; Kubitz, Landers, Petruzzello \& Han, 2007; Youngstedt, O'Connor \& Dishman, 1997). Así mismo, un metaanálisis que tenía como objetivo sintetizar los efectos que tiene la $\mathrm{AF}$ en el sueño, no encontró diferencias significativas entre intensidades (Kredlow, Capazzoli, Hearon, Calkins \& Otto, 2015; Capozzoli, Hearon, Kredlow, Otto \& Calkins, 2015).

Debido a que la información disponible sobre el comportamiento de movimiento de 24 horas (i.e. conducta sedentaria, AF y sueño) medidas de manera objetiva ha sido poco investigada en los estudiantes universitarios de Chile, es que esta investigación tiene como objetivos: 1. describir conducta sedentaria, AF y patrones del sueño en estudiantes de Pedagogía en Educación Física 2. determinar si existen diferencias entre estudiantes según sexo y año que se encuentran cursando, y 3. determinar si existe asociación entre intensidad de AF y patrones del sueño.

\section{Material y método}

Investigación de diseño no experimental, descriptiva, transversal, con un enfoque metodológico cuantitativo.

\section{Sujetos}

Bajo un muestreo no probabilístico se invitó a participar a 250 estudiantes universitarios que cursan Pedagogía en Educación Física, en la ciudad de Temuco, Chile. Se incluyeron aquellos participantes que no presentaron lesiones imposibilitantes, entre 18 y 23 años, y con IMN normal. Se excluyeron quienes no aceptaron $(\mathrm{n}=122)$. La muestra final quedó conformada por 128 estudiantes, dividida de la siguiente forma: primer año con 38 estudiantes (12 mujeres y 26 hombres) con una edad promedio de 18.2 años; segundo año con 30 estudiantes ( 8 mujeres y 22 hombres) con una edad promedio de 19.4 años; tercer año con 40 estudiantes (18 mujeres y 22 hombres) con una edad promedio de 20.1 años; y cuarto año con 20 estudiantes (10 mujeres y 10 hombres) con una edad promedio de 21.4 años. 
La investigación fue apoyada por las autoridades de la Dirección de carrera de Pedagogía en Educación Física. El investigador a cargo se reunió con los estudiantes para proporcionar información sobre los objetivos, métodos del estudio y resolver dudas. Quienes aceptaron participar, firmaron un consentimiento informado en donde se autoriza el uso de la información con fines científicos. La investigación fue desarrollada siguiendo lo expuesto en la declaración de Helsinki.

\section{Instrumentos de medición}

Conducta sedentaria y AF fueron evaluadas objetivamente utilizando un acelerómetro triaxial GENEActiv, modelo Original (ActivInsights Ltd, Cambridgeshire, UK), el cual tiene capacidad de almacenamiento de .5 $\mathrm{Gb}$ en frecuencias de grabación que van de 10 a $100 \mathrm{~Hz}$ y puede recopilar datos por hasta 21 días. La confiabilidad técnica de este acelerómetro fue reportada por Esliger et al. (2011).

El sueño fue medido objetivamente con el mismo instrumento (acelerómetro triaxial GENEActiv, modelo Original), lo cual se ha considerado confiable examinar (te Lindert \& Van Someren, 2013).

\section{Procedimiento}

Para el estudio se tomó una muestra a $100 \mathrm{~Hz}$ y se usó el acelerómetro 7 días consecutivos en la muñeca no dominante. Tras el regreso del dispositivo al centro de coordinación, los datos en bruto se descargaron a archivos «.bin», utilizando epoch de $30 \mathrm{~s}$, a través del software GENEActiv (versión 2.2). Luego se procesaron en R (http: / / cran.r-project.org), utilizando el paquete GGIR (versión 2.0-2). Para determinar conducta sedentaria y AF se utilizaron puntos de corte recomendados para adultos (Esliger et al., 2011). Los parámetros del sueño se calcularon de acuerdo a lo propuesto por Van Hess et al (2015). La información sobre conducta sedentaria y AF de intensidad ligera, moderada y vigorosa, es entregada en minutos promedio por día. En lo que respecta a patrones del sueño, se entregan datos de tiempo en cama, tiempo de sueño y eficiencia del sueño, los dos primeros expresados en minutos promedio por día, mientras que el tercero, el cual corresponde a la proporción entre tiempo en cama y tiempo de sueño, se entrega en porcentaje (\%).

\section{Análisis estadístico}

Para el análisis descriptivo se utilizó promedio y desviación estándar. Para las mujeres, todas las variables fueron sometidas a la prueba de normalidad de
Shapiro-Wilk, mientras que en hombres por medio de Kolmogorov-Smirnov. La comparación de las variables entre año de estudio que se encuentran cursando y sexo, se realizó por medio de un análisis de varianza (ANOVA) de dos vías para grupos independientes y el test de comparaciones múltiples de Scheffe como post hoc, considerando un nivel de significancia de $p<.05$. Además, para analizar la asociación bidireccional entre patrones de sueño y AF se aplicó la técnica de regresión lineal múltiple (método de introducción). Los resultados fueron obtenidos con el paquete estadístico SPSS versión 25.0 .

\section{Resultados}

La Tabla 1 exhibe conducta sedentaria, AF y patrones del sueño de hombres. Destaca que para las tres intensidades de AF, los estudiantes de primer año presentan los valores más altos de minutos promedio por día, los cuales disminuyen progresivamente en quienes tienen más años en la universidad, siendo los que se encuentran cursando cuarto año quienes presentan menor cantidad. Para patrones del sueño, los de cuarto año presentan los promedios por día más altos en tiempo en cama (717.7 min) y tiempo de sueño (493.6 min). Para eficiencia del sueño, los estudiantes de primer año presentan valores más altos $(79.1 \%)$.

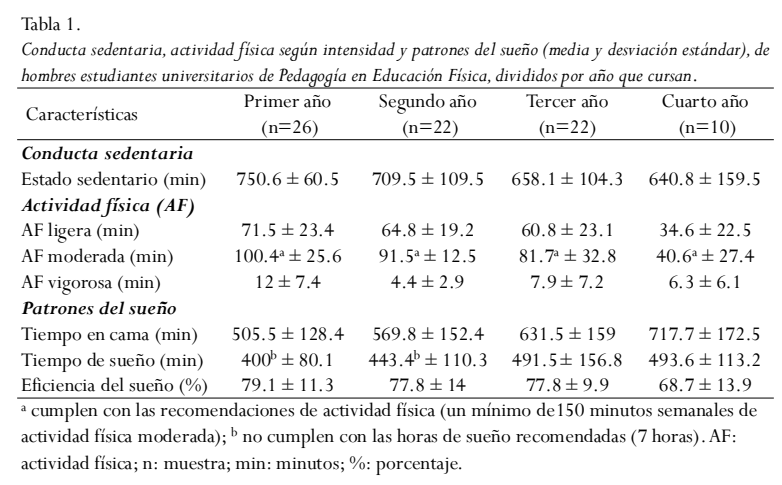

La Tabla 2 presenta conducta sedentaria, AF y patrones del sueño de mujeres. Se observa que las estudiantes de primer año tienen para las tres intensidades de AF, los valores más altos de minutos promedio por día. Para tiempo en cama y tiempo de sueño, los valores más altos son para segundo año (636.5 min y 502.5 min, respectivamente). El porcentaje de eficiencia del sueño es más alto en los estudiantes de primer año (89.8\%).

En relación a las recomendaciones de AF, se evidencia que en promedio todos los estudiantes evaluados, tanto hombres (Tabla 1) como mujeres (Tabla 2), las cumplen. En lo que respecta a horas de sueño para la 
edad, la que corresponde a un mínimo de 7 (Hirshkowitz et al., 2015), hombres de primer y segundo año, $y$ mujeres de primer y tercer año, no las cumplen.

\begin{tabular}{|c|c|c|c|c|}
\hline Características & $\begin{array}{c}\text { Primer año } \\
(\mathrm{n}=12)\end{array}$ & $\begin{array}{c}\text { Segundo año } \\
(\mathrm{n}=8)\end{array}$ & $\begin{array}{c}\text { Tercer año } \\
(\mathrm{n}=18)\end{array}$ & $\begin{array}{c}\text { Cuarto año } \\
(\mathrm{n}=10)\end{array}$ \\
\hline \multicolumn{5}{|l|}{ Conducta sedentaria } \\
\hline Estado sedentario (min) & $771.6 \pm 73.4$ & $676 \pm 83.8$ & $712.8 \pm 69.6$ & $733 \pm 68.5$ \\
\hline \multicolumn{5}{|l|}{ Actividad física $(A F)$} \\
\hline AF ligera (min) & $80.3 \pm 12.1$ & $66.3 \pm 20.8$ & $63.5 \pm 14.5$ & $38.5 \pm 10.5$ \\
\hline AF moderada (min) & $93.8^{a} \pm 23.1$ & $60.7^{\mathrm{a}} \pm 9.6$ & $61.4^{\mathrm{a}} \pm 27.8$ & $40.2^{\mathrm{a}} \pm 22$ \\
\hline AF vigorosa $(\min )$ & $6.5 \pm 3.1$ & $.5 \pm .5$ & $5.7 \pm 5.7$ & $3.6 \pm 3.2$ \\
\hline \multicolumn{5}{|l|}{ Patrones del sueño } \\
\hline Tiempo en cama (min) & $454 \pm 74.2$ & $636.5 \pm 751.1$ & $550.2 \pm 92.6$ & $624.7 \pm 208.6$ \\
\hline Tiempo de sueño (min) & $408.1^{\mathrm{b}} \pm 62.1$ & $502.5 \pm 70.6$ & $443.1^{b} \pm 52.7$ & $450.4 \pm 49.5$ \\
\hline Eficiencia del sueño (\%) & $89.8 \pm 9.8$ & $78.9 \pm 7.6$ & $80.5 \pm 12.3$ & $72 \pm 12.9$ \\
\hline
\end{tabular}

En laTabla 3 se presentan los resultados del ANOVA de dos vías, en donde se comparó cada uno de las características evaluadas, por sexo y año que se encuentran cursando. Se encontró una interacción significativa solo para la variable tiempo en cama. Para el factor año que cursan, se encontraron diferencias estadísticamente significativas en todas las características analizadas. En el factor sexo, se encontró diferencias significativas en el nivel de AF vigorosa y moderada.

\begin{tabular}{|c|c|c|c|}
\hline \multirow[b]{3}{*}{ Características } & \multirow{2}{*}{\multicolumn{3}{|c|}{ Factores }} \\
\hline & & & \\
\hline & $\begin{array}{l}\text { Sexo } \\
F(p)\end{array}$ & $\begin{array}{c}\text { Año que cursan } \\
F(p)\end{array}$ & $\begin{array}{c}\text { Sexo por año que cursan } \\
F(p)\end{array}$ \\
\hline Estado sedentario (min) & $3.5(.063)$ & $4.9(.003)$ & $1.9(.132)$ \\
\hline AF ligera (min) & $1.2(.268)$ & $16.8(<.001)$ & $0.2(.889)$ \\
\hline AF moderada (min) & $9.2(.003)$ & $21.9(<.001)$ & $1.8(.141)$ \\
\hline AF vigorosa (min) & $9.7(.002)$ & $6.3(<.001)$ & $0.5(.682)$ \\
\hline Tiempo en cama (min) & $.3(.551)$ & $18.7(<.001)$ & $2.8(.039)$ \\
\hline Tiempo de sueño (min) & $.1(.735)$ & $4.5(.005)$ & $1.4(.224)$ \\
\hline Eficiencia del sueño (\%) & $1.5(.218)$ & $12.1(<.001)$ & $1.9(.124)$ \\
\hline
\end{tabular}

En el análisis pos-hoc, se encontró que, para estado sedentario, los estudiantes que cursan primer año presentan significativamente menor tiempo sedentario que los otros. No se presentaron diferencias de sexo. Para la variable de AF ligera (Figura 1a) los estudiantes que cursan cuarto año presentan significativamente menor AF ligera en comparación con los otros; mientras que los estudiantes de primer año, presentan significativamente mayor tiempo en comparación con los de tercer año. En relación con la AF moderada (Figura 1b) se encontró que los estudiantes que cursan cuarto año presentan significativamente menor tiempo que los demás, mientras que los de primer año presenta significativamente mayor tiempo que los otros. Por último, se encontró que los estudiantes que cursan primer año realizan significativamente más AF vigorosa que los de segundo y cuarto año; y que los de tercer año presentan mayor AF vigorosa que los de segundo (Figura 1c). Además, se encontró que las mujeres presentan menos AF moderada y vigorosa que los hombres. En la Figura 1 se presenta la comparación de AF y año que cursan.

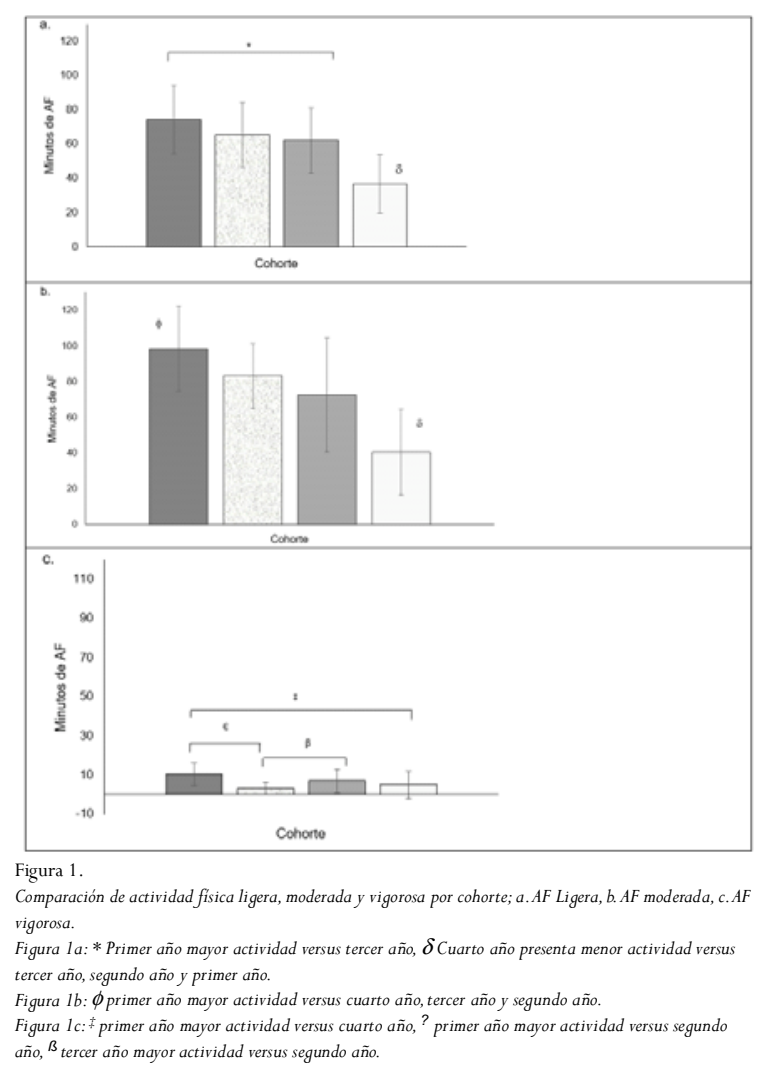

El análisis pos-hoc indicó que, para tiempo en cama, las mujeres presentan mayor tiempo en comparación con los hombres solo en segundo año. En hombres, los estudiantes de cuarto año presentan mayor tiempo en cama en comparación con los demás estudiantes. En las mujeres, las estudiantes de segundo y cuarto año presentan mayor tiempo en cama en comparación con las de primero y tercero. En relación al tiempo de sueño, se encontró que primer año presentó menor tiempo que los demás. Por último, primer año presentó significativamente mayor porcentaje de eficiencia en comparación a los demás estudiantes, y a su vez, cuarto año presenta significativamente menor porcentaje de eficiencia que segundo año.

En la Tabla 4 se muestra la asociación bidireccional analizada por medio de la regresión lineal múltiple. Para predecir la AF ligera a partir de las características del sueño y el sexo, se encontró un modelo significativo ( $F$ $=12.3 ; p<, 001)$, explicando el $26.3 \%\left(R^{2}\right.$ ajustado $)$ de la varianza, ningún factor es significativo. Al predecir la AF moderada a partir de tiempo sueño, tiempo cama, eficiencia y sexo se encontró un modelo significativo $(F$ 
$=14.3 ; p<.001)$, explicando en un $29.5 \%\left(R^{2}\right.$ ajustado) la varianza; el sexo y tiempo en cama son los factores significativos. Por último, al predecir la AF vigorosa se encontró que es un modelo significativo $(F=5.9$; $p$ $<.001)$, explicando un $13.6 \%$ ( $R^{2}$ ajustado) de la varianza, sexo, tiempo de sueño y tiempo en cama son los factores significativos.

Para predecir la eficiencia de sueño a partir de AF ligera, moderada, vigorosa y sexo, se encontró un modelo significativo $(\mathrm{F}=8.3 ; \mathrm{p}<.001)$ que explica el $18.8 \%$ ( $R^{2}$ ajustado) de la varianza, AF ligera es el factor significativo. Para predecir el tiempo en cama a partir de la AF y el sexo, se encontró un modelo significativo $(F=13.2 ; p<.001)$, el modelo explica el $27.8 \%\left(R^{2}\right.$ ajustado) de la varianza, AF ligera es el factor significativo. Por último, se encontró un modelo significativo ( $F$ $=5.8, p<.001)$ para predecir tiempo de sueño a partir de AF, donde el modelo explica el 13.3\% $\left(R^{2}\right.$ ajustado) de la varianza, AF ligera es el factor significativo.

\begin{tabular}{|c|c|c|c|}
\hline $\begin{array}{c}\text { Variable } \\
\text { Dependiente }\end{array}$ & Variables predictoras & $\beta\left(I C_{95 \%}\right)$ & $P$ \\
\hline \multicolumn{4}{|c|}{ Sueño y sexo predicen AF } \\
\hline \multirow{5}{*}{ Ligera } & Constante & $84.8(31 ; 138.7)$ & .002 \\
\hline & Sexo & $-1.3(-8.6 ; .6 .0)$ & .727 \\
\hline & Eficiencia & $.25(-.2 ; .7)$ & .305 \\
\hline & Tiempo cama & $-.04(-.1 ; .1)$ & .089 \\
\hline & Tiempo sueño & $-.04(-.1 ; .1)$ & .093 \\
\hline \multirow{5}{*}{ Moderada } & Constante & $108.7(35.2 ; 182.2)$ & .004 \\
\hline & Sexo & $18.65(8.5 ; 28.7)$ & $<.001$ \\
\hline & Eficiencia & $.03(-.6 ; .7)$ & .911 \\
\hline & Tiempo cama & $-.092(-.2 ;-.3)$ & .004 \\
\hline & Tiempo sueño & $.02(-.1 ; .1)$ & .561 \\
\hline \multirow{5}{*}{ Vigoroso } & Constante & $23.4(6.5 ; 40.4)$ & .007 \\
\hline & Sexo & $2.68(.4 ; 5.0)$ & .023 \\
\hline & Eficiencia & $-.14(-.3 ; .1)$ & .057 \\
\hline & Tiempo cama & $-.02(-.03 ;-.01)$ & .001 \\
\hline & Tiempo sueño & $.02(.01 ; .03)$ & .026 \\
\hline \multicolumn{4}{|c|}{ AF y sexo predicen Sueño } \\
\hline \multirow{5}{*}{ Eficiencia } & Constante & $63.37(56.6 ; 70.1)$ & $\begin{array}{c}<.00 \\
1\end{array}$ \\
\hline & Sexo & $-4.52(-9.3 ; .2)$ & .065 \\
\hline & Ligero & $.13(.0 ; .3)$ & .050 \\
\hline & Moderada & $.12(-.01 ; .24)$ & .052 \\
\hline & Vigoroso & $-.15(-.5 ; .26)$ & .461 \\
\hline \multirow{5}{*}{ Tiempo de cama } & Constante & $852.7(770.6 ; 934.7)$ & $\begin{array}{c}<.00 \\
1\end{array}$ \\
\hline & Sexo & $4.2(-54.2 ; 62.8)$ & .885 \\
\hline & Ligero & $-2.6(-4.3 ;-.9)$ & .002 \\
\hline & Moderada & $-1.21(-2.7 ; .3)$ & .112 \\
\hline & Vigoroso & $-.92(-5.9 ; 4.1)$ & .719 \\
\hline \multirow{5}{*}{ Tiempo de sueño } & Constante & $569.2(514.3 ; 624.1)$ & $\begin{array}{c}<.00 \\
1\end{array}$ \\
\hline & Sexo & $-14.32(-53.4 ; 24.8)$ & .471 \\
\hline & Ligero & $-1.98(-3.1 ;-.1)$ & .001 \\
\hline & Moderada & $.13(-.8 ; 1.1)$ & .789 \\
\hline & Vigoroso & $.69(-2.6 ; 4.0)$ & 685 \\
\hline
\end{tabular}

\section{Discusión}

De acuerdo a la revisión bibliográfica, este estudio es el primero en analizar de manera objetiva, conducta sedentaria, AF según intensidad y patrones del sueño, en estudiantes universitarios de Educación Física en Chile. Los hallazgos más importantes señalan que quienes cursan primer año realizan mayor cantidad de AF en las tres intensidades, además de presentar menor tiempo de sueño, pero una mayor eficiencia. También se encontró que AF de intensidad ligera se asocia con los patrones de sueño evaluados.

Los resultados señalan que, en promedio, todos los evaluados cumplen con las recomendaciones de AF, datos que difieren a lo reportado para población universitaria chilena, donde solo un 16\% de mujeres y un 25\% de hombres cumplen con la AF necesaria para provocar efectos positivos en su salud (Olivares, Lera, \& Bustos, 2008). Lo que sitúa a la población estudiada con mejores parámetros de salud, debido al factor protector que brinda la AF frente a enfermedades crónicas no transmisibles (Fiuza-Luces, Garatachea, Berger, \& Lucia, 2013; Pedersen \& Saltin, 2015).

En lo que respecta a comparaciones, fueron los estudiantes de primer año quienes presentaron menor tiempo de conducta sedentaria y valores más altos para las tres intensidades de AF, situación que ha sido descrita en estudios anteriores (Valdés-Badilla et al., 2013; Vásquez-Gómez et al., 2018), pero que esta vez se corrobora de manera objetiva. Una posible explicación de los valores encontrados puede ser debido a que en primer año, el plan de estudios de la carrera de Pedagogía en Educación Física cuenta con una mayor cantidad de actividades prácticas, lo cual provoca un aumento de la cantidad de AF que realizan; y a medida en que avanzan en los años de estudio se va produciendo un acercamiento al mundo laboral, lo cual provoca una baja de la AF.

La comparación por sexo da cuenta que las mujeres realizan menor cantidad de AF de intensidad moderada y vigorosa, dato que es similar a lo reportado en otras investigaciones (Olivares, Lera, \& Bustos, 2008; Rodriguez-Rodríguez, Cristi-Montero, Villa-González, Solís-Urra \& Chillón, 2018). Sin embargo, para la población evaluada no se consideran tan relevante estas diferencias, debido a que los valores promedios encontrados indican que cumplen con la recomendaciones de AF, pero cobran importancia a futuro, ya que la evidencia disponible expone que mujeres adultas y adultas mayores realizan menor cantidad de AF, debido a factores sociales, fisiológicos y antropométricos (CrespoSalgado, Delgado-Martín, Blanco-Iglesias \& AldecoaLandesa, 2015; González \& Rivas, 2018). Lo cual se convierte en un desafio, para que a futuro las mujeres evaluadas adopten hábitos que les permitan mantener sus niveles de AF, y a quienes se encargan de la salud de la población, implementen medidas para que no se produzca este descenso que afectan en mayor medida a las mujeres.

En lo que respecta a horas de sueño, los valores son 
mejores a lo reportado para población universitaria chilena (Durán-Agüero et al., 2016; Durán et al., 2017), lo cual podría deberse al efecto que ejerce sobre el sueño la AF que realizan, ya que estudios reportan que poblaciones con altos niveles de actividad física presentan mejores parámetros del sueño (Youngstedt \& Kline, 2006; Chennaoui et al., 2015). Al comparar por años que llevan en la universidad, quienes se encuentran en primero, presentan valores más bajos de tiempo de sueño, situación que podría estar relacionada con la mayor cantidad de AF moderada y vigorosa que realizan, ya que como lo exponen Driver \& Taylor (2000), debido al estrés que generan estas intensidades, se provoca una disminución de las horas de sueño. Sin embargo, los mismos estudiantes presentan mayor eficiencia del sueño (i.e. del tiempo que se encuentran en cama, duermen mayor cantidad), situación que se podría relacionar nuevamente con las intensidades de AF que practican en mayor cantidad, ya que también existe evidencia que las respalda con beneficios sobre el tiempo de sueño (Chennaoui et al., 2015; Kelley \& Kelley, 2017). Sin embargo, el análisis de regresión indicó que la AF de intensidad ligera es la que se asocia de manera positiva con los patrones del sueño estudiados, situación que descarta posibles relaciones con AF de intensidad moderada y vigorosa, y que más alla de respaldar a la AF de intensidad ligera como la que entrega los mejores beneficios, aporta información que respalda a lo reportado previamente cuando se expone que los resultados en relación al tema no son concluyentes (Driver \& Taylor, 2000; Kubitz, Landers, Petruzzello \& Han, 2007; Youngstedt et al., 1997; Kredlow, Capazzoli, Hearon, Calkins \& Otto, 2015).

Ante este escenario, y dado que son variados los efectos que tiene la intensidad del ejercicio sobre patrones de sueño, y de acuerdo a lo expuesto por Dolezal, Neufeld, Boland, Martin \& Cooper, (2020) quienes plantean la necesidad de realizar más investigaciones que exploren los mecanismos biológicos que modulan la interacción dinámica entre estos dos aspectos, se hace pertinente en esta población, la realización de ensayos clínicos aleatorizados que permitan determinar con precisión la influencia que están provocando las intensidades de AF sobre patrones del sueño. Lo cual contribuiría a que la población universitaria que no cumple con las recomendaciones de AF y que ve alterado sus patrones del sueño, puedan mejorar por medio de acciones como asignaturas electivas o talleres extra curriculares que incorporen la AF. Se recomienda que futuros estudios incluyan metodologías que permitan determinar la re- lación entre plan de estudios de la carrera de Pedagogía en Educación Física con menor cantidad de AF que se realiza a medida que avanzan en años de estudios. Así como la realización de análisis de composición, lo cual permite centrarse en la investigación del sueño, conducta sedentaria y la AF, interpretando los hallazgos como efectos entre comportamientos, en lugar de que sean independientes (Chastin, Palarea-Albaladejo, Dontje, \& Skelton, 2015).

Este estudio presenta limitaciones que deben ser consideradas en futuras investigaciones, como su diseño transversal, el que no permite hacer inferencias causaefecto; el estudiar una muestra no probabilística, lo que no permite extrapolar los resultados encontrados; el no evaluar algunas variables que pueden influir sobre lo estudiado, como la alimentación, composición corporal y uso de medios electrónicos. Las fortalezas de este estudio están en la objetividad de las mediciones de actividad física y sueño; y la inclusión de variables poco estudiada, lo cual da los primeros pasos para próximas investigaciones.

\section{Conclusiones}

Los resultados indican que los estudiantes de primer año presentan menor tiempo de conducta sedentaria, mayor cantidad de AF en las tres intensidades y menor tiempo de sueño, pero mayor eficiencia. Al comparar por sexo, las mujeres presentan menor cantidad de AF de intensidad moderada y vigorosa. Además, se encontró que AF de intensidad ligera se asoció con los patrones del sueño evaluados. Para una mayor comprensión de la relación intensidad de AF y sueño, se recomienda la realización de ensayos clínicos aleatorios.

\section{Referencias}

Aguilar-Farías, N., \& Leppe, J. (2016). Is a single question of the Global Physical Activity Questionnaire (GPAQ) valid for measuring sedentary behaviour in the Chilean population?. Journal of Sports Sciences, 35(16), 1652-1657. doi: 10.1080/ 02640414.2016.1229010

Almagià, A., Lizana, P., Rodríguez, F., Ivanovich, D., \& Binvignat, O. (2009). Variables Antropométricas y Rendimiento Físico en Estudiantes Universitarios de Educación Física. International Journal of Morphology, 27(4), 971-975. Recuperado de http: / dx.doi.org/ 10.4067/S0717-95022009000400001

Capozzoli, M. C., Hearon, B. A., Kredlow, M. A., Otto, 
M.W., \& Calkins, A.W. (2015).The effects of physical activity on sleep: a meta-analytic review. Journal of Behavioral Medicine, 38(3), 427-449. doi: 10.1007/ s10865-015-9617-6

Chastin, S., Palarea-Albaladejo, J., Dontje, M., \& Skelton, D. (2015). Combined Effects of Time Spent in Physical Activity, Sedentary Behaviors and Sleep on Obesity and Cardio-Metabolic Health Markers: A Novel Compositional Data Analysis Approach. PLos One 10(10), :e0139984. doi: 10.1371/ journal.pone.0139984

Chennaoui, M., Arnal, P. J., Sauvet, F., \& Léger, D. (2015). Sleep and exercise: A reciprocal issue? Sleep Medicine Reviews, 20, 59-72. doi: 10.1016/ j.smrv.2014.06.008

Crespo-Salgado, J., Delgado-Martín, J., Blanco-Iglesias, O., \& Aldecoa-Landesa, S. (2015) Basic guidelines for detecting sedentarism and recommendations for physical activity in primary care. Aten Primaria.47(3), 175-83. doi: 10.1016/j.aprim.2014.09.004

Diekelmann, S., \& Born, J. (2010). The memory function of sleep. Nature Reviews Neuroscience, 11(2), 114-126. doi: $10.1038 / \mathrm{nrn} 2762$

Dolezal, B., Neufeld, E., Boland, D., Martin, J., \& Cooper, C. (2020). Interrelationship between Sleep and Exercise: A Systematic Review. Advances in Preventive Medicine, 1364387. doi: 10.1155/2017/ 1364387

Driver, H. S., \& Taylor, S. R. (2000). Exercise and sleep. Sleep Medicine Reviews, 4(4), 387-402. https:// doi.org/10.1053/smrv.2000.0110

Durán-Agüero, S., Fernández-Godoy, E., Fuentes Fuentes, J., Hidalgo Fernández, A., Quintana Muñoz, C., Yunge Hidalgo, W., ... Delgado Sánchez, C. (2016). Sueño, insomnio y somnolencia en estudiantes, 22(3), 14-19. doi: 10.14642/RENC.2016.22.3.5143

Durán, S., Rosales, G., Moya, C., \& García, P. (2017). Insomnio , latencia al sueño y cantidad de sueño en estudiantes universitarios chilenos durante el periodo de clases y exámenes Salud Uninorte, 33(2), 7585. doi: $10.14482 /$ sun.33.2.10533

Durán, S., Valdés, P., Godoy, A., \& Herrera, T. (2014). Hábitos alimentarios y condición física en estudiantes de pedagogía en educación física. Revista Chilena de Nutrición, 41(3), 251-259. doi: 10.4067/s071775182014000300004

Esliger, D. W., Rowlands, A. V., Hurst, T. L., Catt, M., Murray, P., \& Eston, R. G. (2011). Validation of the GENEA accelerometer. Medicine and Science in Sports and Exercise, 43(6), 1085-1093. doi: 10.1249/

\section{MSS.ObOt3e31820513be}

Fiuza-Luces, C., Garatachea, N., Berger, N.A., \& Lucia, A. (2013). Exercise is the Real Polypill. Physiology, 28(5), 330-358. doi: 10.1152/physiol.00019.2013

Grieser, E. A., Tatum, J. I., Taylor, D. J., Bramoweth, A. D., Williams, J. M., Gardner, C. E., \& Roane, B. M. (2011). Insomnia and Mental Health in College Students. Behavioral Sleep Medicine, 9(2), 107-116. doi: 10.1080/15402002.2011.557992

González, N., \& Rivas, A. (2018). Actividad y ejercicio en mujeres. Revista Colombiana de Cardiología, 25(s1), 125-131. doi: 10.1016/j.rccar.2017.12.008

Hirshkowitz, M., Whiton, K., Albert, S. M., Alessi, C., Bruni, O., DonCarlos, L., ... Adams-Hillard, P. J. (2015). National Sleep Foundation's sleep time duration recommendations: methodology and results summary. Sleep Health, 1(1), 40-43. doi: 10.1016/ j.sleh.2014.12.010

Kelley. G., \& Kelley, K. (2017). Exercise and sleep: a systematic review of previous meta-analyses. J Evid Based Med, 10(1), 26-36. Doi: 10.1111/jebm.12236

Kredlow, M., Capozzoli, M., Hearon, B., Calkins, A., \& Otto, M. (2015). The effects of physical activity on sleep: a meta-analytic review. J Behav Med, 38(3), 427-29. doi: 10.1007/s10865-015-9617-6

Kubitz, K.A., Landers, D. M., Petruzzello, S. J., \& Han, M. (2007). The Effects of Acute and Chronic Exercise on Sleep. Sports Medicine, 21(4), 277-291. doi : 10.2165/00007256-199621040-00004

Lazar, A. S., von Schantz, M., Arbon, E. L., Groeger, J. A., Lo, J. C., Santhi, N., ... Dijk, D.J. (2012). Effects of Partial and Acute Total Sleep Deprivation on Performance across Cognitive Domains, Individuals and Circadian Phase. PLoS ONE, 7(9), e45987. doi: 10.1371 /journal.pone.0045987

Lund, H. G., Reider, B. D., Whiting, A. B., \& Prichard, J. R. (2010). Sleep Patterns and Predictors of Disturbed Sleep in a Large Population of College Students. Journal of Adolescent Health, 46(2), 124-132. doi: 10.1016/j.jadohealth.2009.06.016

Olivares C, S. , Lera M, L., \& Bustos Z, N. (2008). Stages of change, benefits and barriers on physical activity and fruit and vegetables intake in Chilean university students. Revista Chilena de Nutricion, 35(2), 25-35. Recuperado de https: / /www.scopus.com/inward/ r e c o r d . u r i ? e i d = 2-s 2 . 0 46449109103\&partnerID $=40 \& \mathrm{md} 5=\mathrm{e} 8 \mathrm{f} 604 \mathrm{ce} 78$ 4b8fb8d580946fcd68a8df\%0Ahttps: / /

Organización Mundial para la Salud, O. (2020). Directrices de la OMS sobre actividad física y hábitos se- 
dentarios. Organización Mundial Para La Salud. Recuperado de https://www.who.int/es/activities/ preventing-noncommunicable-diseases / 9789240014886

Pedersen, B. K., \& Saltin, B. (2015). Exercise as medicine - Evidence for prescribing exercise as therapy in 26 different chronic diseases. Scandinavian Journal of Medicine and Science in Sports, 25, 1-72. doi: $10.1111 /$ sms. 12581

Peltzer, K., \& Pengpid, S. (2015). Nocturnal sleep problems among university students from 26 countries. Sleep and Breathing, 19(2), 499-508. doi: 10.1007/s11325-014-1036-3

Rodríguez-Rodríguez, F., Cristi-Montero, C., VillaGonzález, E., Solís-Urra, P., \& Chillón, P. (2018). Comparación de los niveles de actividad física durante la vida universitaria. Rev Med Chil, 146(4), 442 450. doi: 10.4067/s0034-98872018000400442

te Lindert, B. H. W., \& Van Someren, E. J. W. (2013). Sleep Estimates Using Microelectromechanical Systems (MEMS). Sleep, 36(5), 781-789. doi: 10.5665 /sleep. 2648

Valdés-Badilla, P., Godoy-Cumillaf, A., \& Gedda-Muñoz, R. (2013). Comparación por cohorte de la condición física de estudiantes de pedagogía en educación física de la Universidad Autónoma de Chile, sede Temuco., 4, 76-85. Recuperado de http:// revistahorizonte.ulagos.cl/index.php/horizonte/ article/view/49

Valdés-Badilla, P., Godoy-Cumillaf, A., HerreraValenzuela, T., \& Durán-Agüero, S. (2015). The comparison between food habits and physical condition among physical education and other undergraduate students. Nutricion Hospitalaria, 32(2). doi: 10.3305/ nh.2015.32.2.9194

Vásquez-Gómez, J. A., Castillo-Retamal, M. E., de Carvalho, R., Faundez-Casanova, C. P., TorrealbaCampos, A. P., Vásquez-Gómez, J. A., ... TorrealbaCampos, A. P. (2018). Antropometría, nivel de actividad física y condición física en estudiantes de educación física tras cuatro años en la universidad]. Nutricion Clinica y Dietetica Hospitalaria, 38(1), 160 164. doi: 10.12873/381JVasquez

Van Hess, V., Sabia, S., Andersen, K., Denton, S., Oliver, J., Catt, M., ... Singh-Manoux,A. (2015). Novel Open Access Method to Assess Sleep Duration Using a Wrist-Worn Accelerometer. PLos One, 16(10), e0142533. doi: 10.1371/journal.pone.0142533

Vélez, J., Souza, A., Traslaviña, S., Barbosa, C., Wosu, A., Andrade, A., ... Williams, M. (2013). The Epidemiology of Sleep Quality and Consumption of Stimulant Beverages among Patagonian Chilean College Students. Sleep Disorders, 910104. doi: 10.1155/2013/910104

Wolfson, A. R. (2010). Adolescents and Emerging Adults' Sleep Patterns: New Developments. Journal of Adolescent Health, 46(2), 97-99. doi: 10.1016/ j.jadohealth.2009.11.210

Youngstedt, S. D., \& Kline, C. E. (2006). Epidemiology of exercise and sleep. Sleep and Biological Rhythms, $4(3), \quad 215-221$ doi: $10.1111 / \mathrm{j} .1479$ 8425.2006.00235.x

Youngstedt, S. D., O'Connor, P., \& Dishman, R. (1997). The effects of acute exercise on sleep: a quantitative synthesis. Sleep, 20(3), 203-14. doi: 10.1093/sleep/ 20.3.203

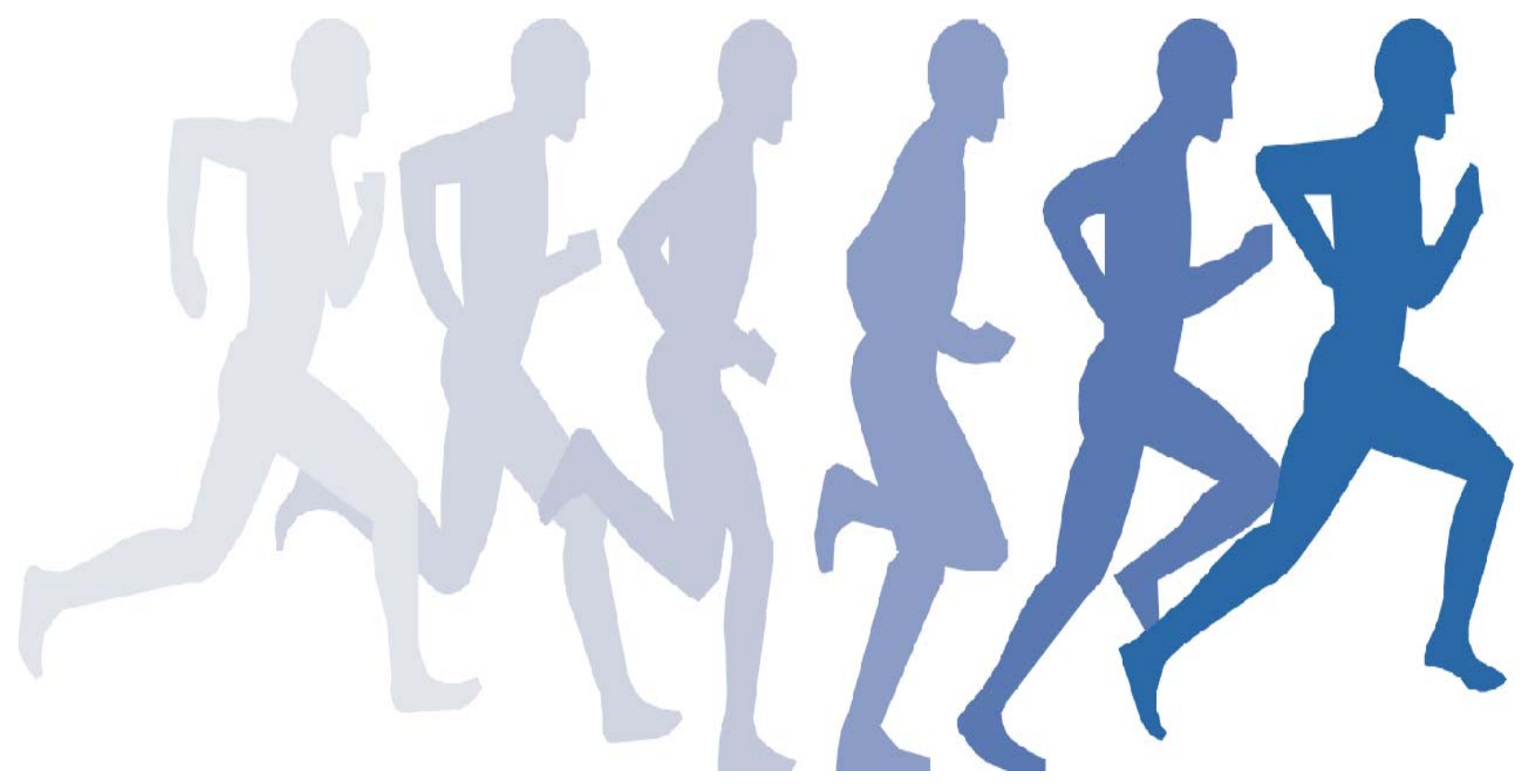

